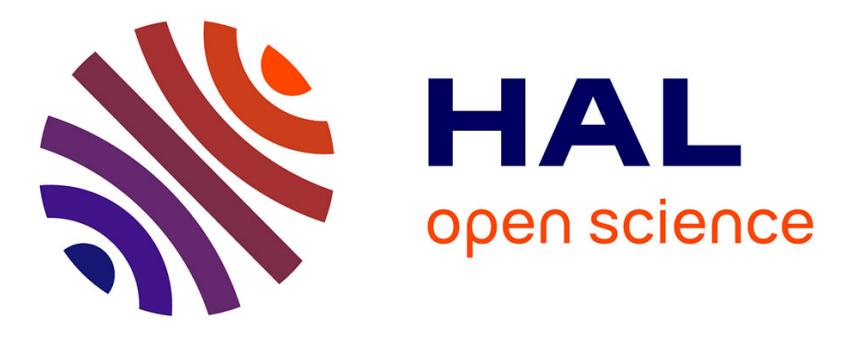

\title{
Model Predictive Control With Obstacle Avoidance for Inertia Actuated AFM Probes Inside a Scanning Electron Microscope
}

\author{
Shuai Liang, Mokrane Boudaoud, Pascal Morin, Jonathan Cailliez, \\ Barthélemy Cagneau, Weibin Rong, Stéphane Régnier
}

\section{To cite this version:}

Shuai Liang, Mokrane Boudaoud, Pascal Morin, Jonathan Cailliez, Barthélemy Cagneau, et al.. Model Predictive Control With Obstacle Avoidance for Inertia Actuated AFM Probes Inside a Scanning Electron Microscope. IEEE Robotics and Automation Letters, 2021, 6 (2), pp.382 - 388. 10.1109/LRA.2020.2974388 . hal-03115963

\section{HAL Id: hal-03115963 \\ https://hal.science/hal-03115963}

Submitted on 19 Jan 2021

HAL is a multi-disciplinary open access archive for the deposit and dissemination of scientific research documents, whether they are published or not. The documents may come from teaching and research institutions in France or abroad, or from public or private research centers.
L'archive ouverte pluridisciplinaire HAL, est destinée au dépôt et à la diffusion de documents scientifiques de niveau recherche, publiés ou non, émanant des établissements d'enseignement et de recherche français ou étrangers, des laboratoires publics ou privés. 
Model Predictive Control With Obstacle Avoidance for Inertia Actuated AFM Probes Inside a Scanning Electron Microscope

\author{
Shuai Liang ${ }^{\circledR}$, Mokrane Boudaoud, Pascal Morin, Jonathan Cailliez, Barthelemy Cagneau ${ }^{\circledR}$, \\ Weibin Rong, and Stephane Régnier
}

\begin{abstract}
The Atomic Force Microscope (AFM) is a reliable tool for 3D imaging and manipulation at the micrometer and nanometer scales. When used inside a Scanning Electron Microscope (SEM), AFM probes can be localized and controlled with a nanometer resolution by visual feedback. However, achieving trajectory control and obstacles avoidance is still a major concern for manipulation tasks. We propose a Model Predictive Control (MPC) to address these two issues while AFM probes are actuated by Piezoelectric Inertia type Actuators (PIA). The novelty of this letter is that the model of our MPC-based approach relies on a velocity map of PIAs. It enables path following and obstacle avoidance while preserving safety margins. Control inputs are optimized by Quadratic Programming, referring to their increment and distance constraints. A cost function is defined to navigate the AFM probe with a specified velocity. Simulations and experiments are carried out to demonstrate that the proposed algorithm is suitable to perform path following with obstacle avoidance using map-based velocity references. This is the first time that MPC is implemented in micro/nano-robotic systems for autonomous control inside SEM.
\end{abstract}

Index Terms: Atomic Force Microscopy, Robot Control, Path planning.

\section{INTRODUCTION}

$\mathbf{N}$ ANO-ROBOTIC systems operating inside Scanning Electron Microscopes (SEM) have become key tools to face the current challenges in micro/nano technologies for both industry and academic research [1]. They have been applied in a wide range of emerging domains, such as nano-material characterization [2], [3], nano-assembly [4], [5] and dual SEM/AFM imaging [6]. The choice of using SEM is mainly motivated by some of its performance that can not be achieved with classical

Manuscript received September 10, 2019; accepted January 16, 2020. Date of publication; date of current version. This letter was recommended for publication by Associate Editor W. Wang and Editor A. Okamura upon evaluation of the reviewers' comments. This work was supported by the Research Program ANR JCJC Robine. (Corresponding author: Shuai Liang.)

Shuai Liang, Mokrane Boudaoud, Pascal Morin, Jonathan Cailliez, and Stephane Régnier are with the Sorbonne Universités, F-75005 Paris, France (e-mail: liang@sorbonne-universite.fr; mokrane.boudaoud @ sorbonne-universite.fr; morin@ sorbonne-universite.fr; cailliez@ isir.upmc.fr; stephane.regnier@sorbonne-universite.fr).

Barthelemy Cagneau is with the Université de Versailles Saint-Quentin-enYvelines, Laboratoire d'Ingénierie des Systèmes de Versailles (LISV), 78035 Versailles cedex, France (e-mail: barthelemy.cagneau@uvsq.fr).

Weibin Rong is with the Harbin Institute of Technology, State Key Lab. of Robotics and System, Harbin 150080, China (e-mail: rwb@hit.edu.cn). optical microscopes such as the nano-metric imaging resolution and the depth of field which is necessary to simultaneously observe robots end effectors and samples.

Works in the literature have tackled critical issues of robotics inside SEMs. The degree of automation can be categorized into three levels: low-automated, semi-automated and fullyautomated. The low-automated level control is based on teleoperated systems which performances strongly depend on user skills and available information [7], [8]. The process is timeconsuming and often not repeatable because of the user-centered approach. The semi-automated level provides the user with enhanced information even for non-observable environments (e.g. vision, sound and touch). Virtual Reality (VR) is a well-known example of such strategies [9], [10]. In other applications, the task might be divided between automated and manual process [11] while other strategies automatically repeat a manually pre-defined sequence [12], [13], [14], [15]. The fully automated level control makes sense if high success rate can be achieved instead of one-shot operated tasks. Therefore, it is often performed with specific conditions and/or with dedicated tools [4]. For example, in [16], given an initial random configuration, nanoparticles are organized into desired patterns. However, the method is not robust against actuators model uncertainties since an accurate model is required to compensate for drift, creep and hysteresis effects. In [17], authors explain why combining AFM and SEM would be a convenient solution to manipulate objects while imaging. However, again, specific modes of operation or dedicated sensors and actuators limit its development for fully automated tasks. Regarding the literature, it is thus still challenging to complete advanced automated tasks without the need of specific conditions or tools.

This letter targets the development of a user-friendly interface that can manage elementary robotic tasks needed inside a SEM by simply defining graphical instructions with a mouse click on the SEM screen. This capability can be included into a human/robot semi-automated control. More specifically, we target the design of a SEM vision based path following control strategy with a Model Predictive Controller (MPC) for nano-robot end effectors with obstacle avoidance capabilities. Since SEM provides a week depth information, obstacle avoidance in needed to avoid damaging the robot end effector when moving across areas with several objects located along its desired trajectory. Such objects can be surrounded by restricted zones drawn by the user on the SEM screen. These zones are considered as obstacles that the end effector must avoid. In this work, the end effector is a self-sensing Atomic Force Microscope (AFM) probe. The 
AFM operates in micrometer and nanometer sized areas when working around the target area for topography applications or micro/nano-manipulation. However, to bring the AFM probe into these target areas, it requires a long range positioning. Particularly, when used inside SEM, the long range positioning can reach several millimeters. For this reason, the AFM probe is mounted on a 3 axes Cartesian nanorobot actuated by Piezoelectric Inertia Actuators (PIAs) as it enables motions in the centimeter range with a nanometer resolution [18], [19], [20].

The objective of the controller is twofold. When no obstacle is in the neighborhood of the path, a path following control algorithm without prediction capabilities is sufficient. However, when it comes to avoid an obstacle, the MPC is of importance. The latter uses an internal model of the process to predict its future states. This prediction is needed to be able to adjust the dynamics of the robotic system (i.e. controlled energy reduction) when approaching the obstacle and to avoid it in a secure way. The novelty of this letter is that the model of our MPC-based approach relies on a velocity map of PIAs. It enables path following and obstacle avoidance while preserving safety margins. Control inputs are optimized by Quadratic Programming, referring to their increment and distance constraints. A cost function is defined to navigate the AFM probe with a specified velocity.

The paper is organized as follows. Section II recalls some basis of MPC. The experimental platform as well as the characterization of the velocity map of PIAs are described in section III. The control strategy is detailed in section IV-VI. Simulations and experiments are carried out in section VII to demonstrate the effectiveness of the proposed algorithm.

\section{Model Predictive Control}

Model Predictive Control (MPC) is one of the most popular technic to achieve path following with obstacle avoidance ability. This method relies on an internal dynamic model of the controlled process to anticipate its future behavior. One of the main advantages of this control method is that it can anticipate future events and then adapt the control law accordingly.

The use of an internal model of the process is then fundamental for MPC. In the case of PIAs, we have proposed in [18] a comprehensive model of such class of actuators. These actuators include several dynamics such as those of a piezoelectric element and those of the presiding caused by friction. Due to the high resonance frequency of the piezoelectric element (around $64 \mathrm{kHz}$ and $118 \mathrm{kHz}$ ), this model requires a high sampling frequency for its real time implementation. Therefore, we propose here a novel idea for the design of MPC controller in the case of PIAs based on a velocity map easy to implement in real time. PIAs are actuated by sawtooth type voltages. The velocity of the actuator depends on the amplitude and the frequency of the sawtooth input voltage as well as its slope at each period. This slope also defines the direction of motion of the actuator. See [18] and [21] for more details about the working principle and velocity properties of PIAs.

\section{ISIR-RoBOTEX NANO-ROBOTIC PLATFORM AND VELOCITY CHARACTERIZATION}

The parts of the ISIR-robotex nano-robotic platform [18] that will be used in this letter are composed of (Fig. 1) a 3 axes XYZ Cartesian nano-robotic system actuated by PIA of type stick-slip (SLC-1720), a SEM (ZEISS EVO LS 25) and a controller board.

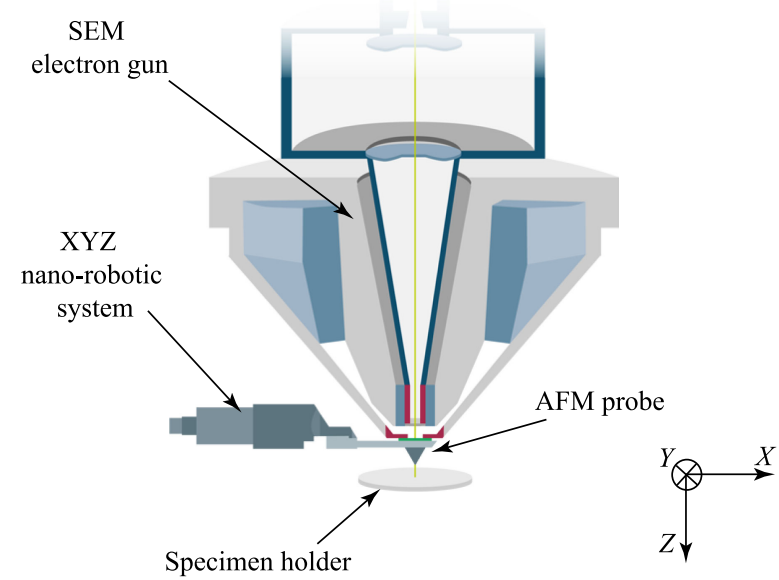

Fig. 1. Dual SEM/AFM schematic view.

The nano-robot operates inside the SEM which provides visual feedback. The controller board (dSPACE DS1007) and a control interface (dSPACE ControlDesk) are used for control algorithms implementation to drive the nano-robotic system. The $\mathrm{Z}$ axis of the nano-robot holds a self-sensing AFM probe.

The obstacles can be identified with the SEM vision. Several SEM scanning speeds are available from scan mode 1 to scan mode 9. The scan mode 1 is the fastest one. It allows obtaining an image every $1.3 \mathrm{~s}$, but at the price of a limited image resolution. A better image quality can be obtained with scan mode 5 , but the acquisition time increases to $15 \mathrm{~s}$. Thus, the real-time capability to identify obstacles depends on a tradeoff between the image quality and the scan speed. In addition, in the working conditions of the paper, the obstacles are static. It is not necessary to identify them at each sampling time.

Experiments in [21] have illustrated that the velocity of PIAs is dependent on the direction, the amplitude and the frequency of the driving sawtooth input voltage. Here a velocity map of PIAs is characterized in a low velocity range, i.e. $<25 \mu \mathrm{m} / \mathrm{s}$, which has not been performed in previous works.

The experiments are repeated for sawtooth voltages with the following frequencies: $1 \mathrm{~Hz}, 3 \mathrm{~Hz}, 5 \mathrm{~Hz}, 7 \mathrm{~Hz}, 9 \mathrm{~Hz}, 11 \mathrm{~Hz}$, $13 \mathrm{~Hz}, 15 \mathrm{~Hz}, 17 \mathrm{~Hz}, 19 \mathrm{~Hz}, 20 \mathrm{~Hz}$. For each frequency, different amplitudes are applied, namely $35 \mathrm{~V}, 40 \mathrm{~V}, 60 \mathrm{~V}, 80 \mathrm{~V}, 90 \mathrm{~V}$. For each operating point, the average velocity is calculated as in [21]. The velocity map of $\mathrm{x}$ and $\mathrm{y}$ axes are fitted by a polynomial interpolation. For instance, Fig. 2 shows the identified velocity map of y axis. The max velocities are $17.23 \mu \mathrm{m} / \mathrm{s}$ and $-22.96 \mu \mathrm{m} / \mathrm{s}$ for forward and backward directions respectively.

Due to the friction force, the PIAs have a dead zone below $10 \mathrm{~V}$. We have selected $35 \mathrm{~V}-90 \mathrm{~V}$ to be well above this dead-zone. Below $35 \mathrm{~V}$, the velocity is considered equal to zero. The velocity map is used in the sequel for plant states estimation and for MPC algorithm optimization.

\section{NOTATION AND MODELING}

The ISIR-robotex platform is modeled as a holonomic system moving in the plane with fixed orientation. The objective is to make the platform follow a given reference path, while avoiding obstacles. These obstacles are assumed to be fixed. The proposed approach is described in the case of a single obstacle. It can be 


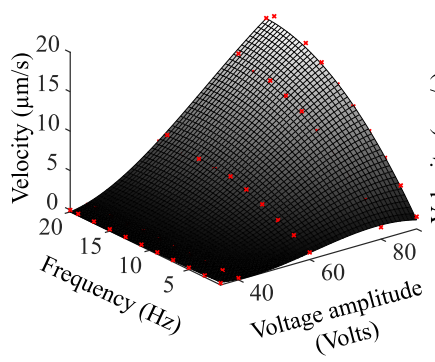

(a)

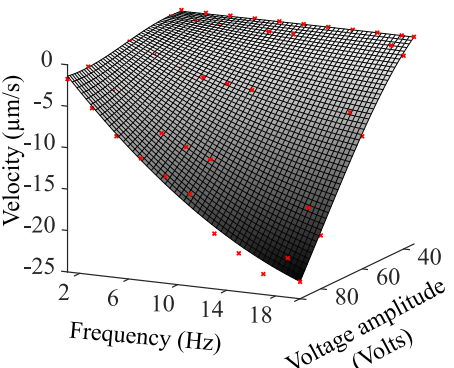

(b)
Fig. 2. Identified velocity maps on Y axis: forward direction (left) and backward direction (right).

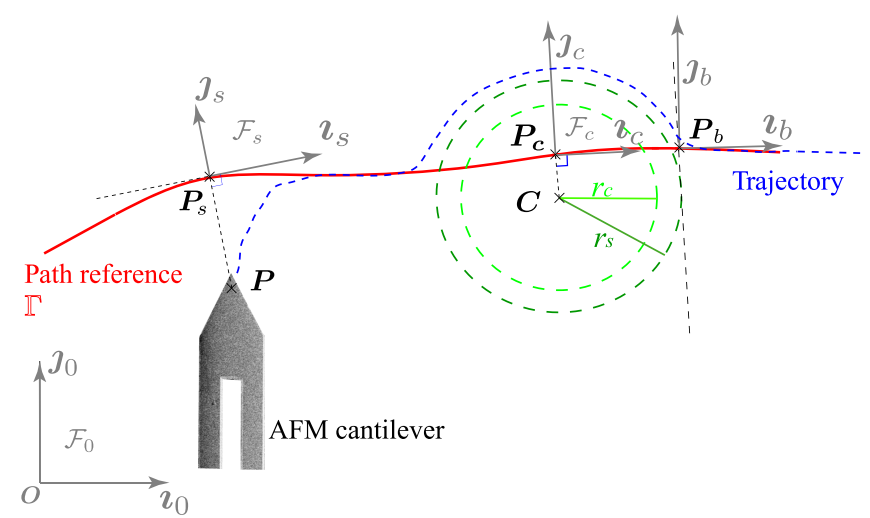

Fig. 3. Kinematic model for model predictive control and obstacle avoidance.

applied in the presence of several obstacles provided the obstacles are separated enough so that the safety discs surrounding each obstacle (as defined below) do not intersect. The following notation is used, in accordance with Fig. 3.

- Points in Euclidean space are denoted with bold uppercase letters. Coordinates of points with respect to a frame are denoted with ordinary (i.e., non-bold) lowercase letters.

- Vectors in Euclidean space are denoted with bold lowercase symbols. Coordinates of vectors w.r.t. (with respect to) a frame are denoted with ordinary letters.

- $\mathcal{F}_{0}=\left\{\boldsymbol{O}, \boldsymbol{\imath}_{0}, \boldsymbol{\jmath}_{0}\right\}$ is a fixed (inertial) frame.

- $\Gamma$ is the reference path.

- $\boldsymbol{P}$ is the point to be controlled, which corresponds to the AFM probe tip. The vector of coordinates of $\boldsymbol{P}$ w.r.t. $\mathcal{F}_{0}$ is denoted as $p=\left[p_{x}, p_{y}\right]^{T}$.

- $\mathcal{F}_{s}=\left\{\boldsymbol{P}_{s}, \boldsymbol{\imath}_{s}, \boldsymbol{J}_{s}\right\}$ is the Frenet frame of $\Gamma$ with origin $\boldsymbol{P}_{s}$ given by the orthogonal projection of $\boldsymbol{P}$ onto the reference path $\Gamma$. The vector of coordinates of $\boldsymbol{P}_{s}$ w.r.t. $\mathcal{F}_{0}$ is denoted as $p_{s}=\left[p_{s_{x}}, p_{s_{y}}\right]^{T}$.

- The obstacle is modeled by a covering disc of radius $r_{c}$ centered at point $\boldsymbol{C}$. The vector of coordinates of $\boldsymbol{C}$ w.r.t. $\mathcal{F}_{0}$ is denoted as $c=\left[c_{x}, c_{y}\right]^{T} . \mathcal{F}_{c}=\left\{\boldsymbol{P}_{\boldsymbol{c}}, \boldsymbol{\imath}_{c}, \boldsymbol{J}_{c}\right\}$ is the Frenet frame of $\Gamma$ with origin $\boldsymbol{P}_{c}$ given by the orthogonal projection of $C$ onto the reference path $\Gamma$. The vector of coordinates of $\boldsymbol{P}_{c}$ w.r.t. $\mathcal{F}_{0}$ is denoted as $p_{c}=\left[p_{c_{x}}, p_{c_{y}}\right]^{T}$.

- A so-called "safety radius" is defined as the radius $r_{s}>r_{c}$ of a circle centered at $C$.

- $l_{\text {border }}$ is the line that is tangent to the circle centered at $C$ of radius $r_{s}$, parallel to $\boldsymbol{J}_{c}$, and intersects the axis $\left\{\boldsymbol{P}_{\boldsymbol{c}}, \boldsymbol{\imath}_{c}\right\}$ at a positive abscissa along this axis. It is treated as the terminating border of the obstacle, after which the obstacle avoidance is considered as complete. The intersection point of $l_{\text {border }}$ with $\Gamma$ is denoted as $\boldsymbol{P}_{b}$ and to this point we associate a frame $\left\{\boldsymbol{P}_{b}, \boldsymbol{\imath}_{b}, \boldsymbol{J}_{b}\right\}$ with $\boldsymbol{\jmath}_{b}$ parallel to $\boldsymbol{J}_{c}$.

The kinematic model is simply given by:

$$
\dot{p}=v=\left[v_{x}, v_{y}\right]^{T}
$$

where $v_{h}$ for $h \in\{x, y\}$ is defined as:

$$
v_{h}=\left\{\begin{array}{l}
v_{h}^{+}=F_{h}^{+}\left(a_{h}, f_{h}\right) \text { for forward motion } \\
v_{h}^{-}=F_{h}^{-}\left(a_{h}, f_{h}\right) \text { for backward motion }
\end{array}\right.
$$

Here $F_{h}^{+}$and $F_{h}^{-}$are the velocity maps discussed in the previous section, with $F_{y}^{+}$and $F_{y}^{-}$depicted on Fig. 2, and $a_{h}, f_{h}$ respectively denote the amplitude and frequency of the sawtooth input voltage.

\section{MPC ALGORITHM}

An MPC algorithm is used for planning and control. We provide below the discrete-time dynamic equations of the AFM probe tip that are used to define the MPC strategy. In the sequel, for any time-function $\xi$ and any integer $k, \xi_{\mid k}=\xi\left(k T_{h}\right)$ where $T_{h}$ is a sampling period. Using an explicit Euler scheme,(1) is discretized as follows:

$$
p_{\mid k+1}=p_{\mid k}+T_{h} v_{\mid k}
$$

For $h \in\{x, y\}$ and $d_{h} \in\{+,-\}$, the function $v_{h}^{d_{h}}$ in (2) is discretized as follows:

$$
\begin{aligned}
v_{h \mid k+1}^{d_{h}}=v_{h \mid k}^{d_{h}} & +\frac{\partial F_{h}^{d_{h}}}{\partial a_{h}}\left(a_{h \mid k}, f_{h \mid k}\right) \Delta a_{h \mid k} \\
& +\frac{\partial F_{h}^{d_{h}}}{\partial f_{h}}\left(a_{h \mid k}, f_{h \mid k}\right) \Delta f_{h \mid k}
\end{aligned}
$$

where the amplitude increments $\Delta a_{h \mid k}=a_{h \mid k+1}-a_{h \mid k}$ and frequency increments $\Delta f_{h \mid k}=f_{h \mid k+1}-f_{h \mid k}$ of the sawtooth voltages are the discrete-time control variables of the MPC algorithm. From (3) and (4), one obtains for each "motion direction" $d=\left(d_{x}, d_{y}\right)$ with $d_{x}, d_{y} \in\{+,-\}$ a discrete-time dynamic model of the AFM probe tip defined as follows:

$$
\left\{\begin{array}{l}
X_{k+1}=A X_{k}+B_{k}^{d} U_{k} \\
Y_{k}=C X_{k}
\end{array}\right.
$$

with state vector $X_{k}$, input $U_{k}$ and output $Y_{k}$ defined as:

$$
\left\{\begin{array}{l}
X_{k}=\left[p_{\mid k}^{T}, v_{\mid k}^{T}, a_{x \mid k}, f_{x \mid k}, a_{y \mid k}, f_{y \mid k}\right]^{T} \\
Y_{k}=\left[p_{\mid k}^{T}, v_{\mid k}^{T}\right]^{T} \\
U_{k}=\left[\Delta a_{x \mid k}, \Delta f_{x \mid k}, \Delta a_{y \mid k}, \Delta f_{y \mid k}\right]^{T}
\end{array}\right.
$$

and state matrices $A, B_{k}^{d}$, and $C$ given by:

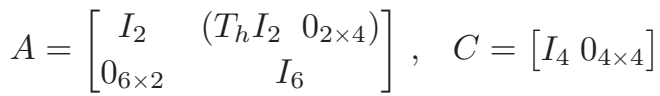

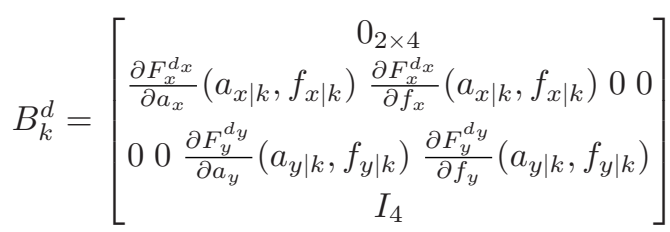


where $0_{m \times n}$ denotes the $m \times n$ zero matrix and $I_{m}$ the $m \times m$ identity matrix. Note that $A$ and $C$ are constant matrices, i.e., they depend neither on the sampling time nor on the motion direction. In contrast, $B_{k}^{d}$ depends on both the sampling time and the motion direction.

For a given cost function, at each sampling time $k T_{h}$ the MPC strategy takes into account the process model (5) to optimize a sequence of control inputs over a prediction horizon $N T_{h}$. Only the first input value $U_{k}$ is applied and this process is iterated at the following sampling times $(k+1) T_{h},(k+2) T_{h}, \ldots$ Let

$$
\bar{Y}_{k}=\left[\begin{array}{c}
Y_{k+1} \\
Y_{k+2} \\
\vdots \\
Y_{k+N}
\end{array}\right], \bar{X}_{k}=\left[\begin{array}{c}
X_{k} \\
X_{k+1} \\
\vdots \\
X_{k+N-1}
\end{array}\right], \bar{U}_{k}=\left[\begin{array}{c}
U_{k} \\
U_{k+1} \\
\vdots \\
U_{k+N-1}
\end{array}\right]
$$

Then, one verifies that

$$
\bar{Y}_{k}=\bar{A}_{k} \bar{X}_{k}+\bar{B}_{k}^{d} \bar{U}_{k}
$$

with

$$
\begin{aligned}
\bar{A}_{k} & =\left[\begin{array}{c}
C A \\
C A^{2} \\
\vdots \\
C A^{N}
\end{array}\right] \\
\bar{B} &
\end{aligned}
$$

Let

$$
\bar{Y}_{\star \mid k}=\left[\begin{array}{c}
Y_{\star \mid k+1} \\
Y_{\star \mid k+2} \\
\vdots \\
Y_{\star \mid k+N}
\end{array}\right], \bar{Y}_{d \mid k}=\left[\begin{array}{c}
Y_{d \mid k+1} \\
Y_{d \mid k+2} \\
\vdots \\
Y_{d \mid k+N}
\end{array}\right]
$$

with

$$
Y_{\star \mid k+i}=\left[\begin{array}{c}
p_{\mid k+i} \\
V_{\mid k+i}
\end{array}\right], Y_{d \mid k+i}=\left[\begin{array}{c}
p_{s_{\mid k+i}} \\
V_{d \mid k+i}
\end{array}\right] \quad(i=1, \ldots, N)
$$

Here $V=\|v\|$ and $V_{d} \geq 0$ is a desired velocity, which will usually be specified by the user. Note that $Y_{\star}$ can be easily computed from $Y$. The objective of the path following problem is to stabilize $\boldsymbol{P}$ to $\boldsymbol{P}_{s}$ (i.e., make the AFM probe tip track its projection $\boldsymbol{P}_{s}$ onto the path $\Gamma$ ) while ensuring a motion speed of the tip equal to $V_{d}$. In other words, the objective is to make $Y_{\star}$ converge to $Y_{d}$. To solve this problem, the MPC algorithm computes $\bar{U}_{k}^{\star}$ as follows:

$$
\overline{U_{k}^{\star}=} \underset{\begin{array}{c}
\bar{U}_{k} \\
\text { such that }
\end{array}}{\arg \min _{i=1}}\left\|Y_{\star \mid k+i}-Y_{d \mid k+i}\right\|_{w}{ }^{2}
$$

where $\|\xi\|_{w}^{2}=\sum_{i=1}^{3} w_{i} \xi_{i}^{2}, \forall \xi \in \mathbb{R}^{3}$, for some weights $w_{1}, w_{2}, w_{3}$.
Quadratic Programming (QP) is used to calculate the optimal $U_{k}^{\star}$. The set of constraints contains both input constraints and state constraints. The input constraints are defined as follows:

$$
\forall k, \forall j=1, \ldots, 4, \quad U_{j, \min } \leq U_{k, j} \leq U_{j, \max }
$$

The state constraints are defined as follows:

$$
\begin{gathered}
\forall k, \forall h \in\{x, y\}, \quad a_{h, \text { min }} \leq a_{h, k} \leq a_{h, \text { max }} \\
\forall k, \forall h \in\{x, y\}, \quad f_{h, \text { min }} \leq f_{h, k} \leq f_{h, \text { max }} \\
\forall k, \quad \rho_{k} \geq r_{s}
\end{gathered}
$$

with $\rho_{k}=\left\|p_{\mid k}-c\right\|$ denoting the distance between the AFM probe tip and the center of the obstacle at sampling time $k T_{h}$.

\section{Two LAYERS CONTROL SCHEME}

The discrete-time dynamic model (5) is a standard linear model provided the direction vector $d$ is constant. In this case, one can make use of standard techniques to solve at each sampling time the MPC optimization problem, as explained in the previous section. Changing the motion direction of the robot may require to change the direction vector, however, so that a strategy is needed at this level. In this work, a two-layers control structure is proposed, customizing the direction-dependency of PIA (Fig. 4). Firstly the high-level layer determines the components $d_{x}, d_{y}$ of the AFM probe direction vector. Secondly, according to the selected velocity maps, the low-level layer executes the MPC optimization as described in the previous section. Following [22], a varying sampling period $T_{h}$ is used, with $T_{h}^{\min } \leq T_{h} \leq T_{h}^{\max }$. Indeed, during obstacle avoidance a longer time horizon interval is beneficial to pre-view further interaction between the AFM probe and the obstacle. The choice of both the direction vector and sampling period depends on the location of the AFM probe tip with respect to the obstacle. To present the proposed strategy, some additional notation is needed. In addition to the circles centered at $\boldsymbol{C}$ of radius $r_{c}$ and $r_{s}$, we define two other circles centered at $\boldsymbol{C}$, or radius $r_{h, 1}$ and $r_{h, 2}$ with $r_{h, 2}=2 r_{c}<r_{h, 1}=3 r_{c}$. Finaly, we denote as $\gamma$ the vector from $\boldsymbol{P}$ to the orthogonal projection of $\boldsymbol{P}$ onto the line $l_{\text {border }}$. From there, $d_{x}, d_{y}$, and $T_{h}$ are chosen as follows:

Case 1: $\rho_{k}>r_{h, 1}$ or $\gamma \cdot \boldsymbol{\imath}_{b} \leq 0$. These conditions imply that the collision risk is low. Then we set:

$d_{x}=\operatorname{sign}\left(\left(\boldsymbol{\imath}_{s}+\delta \boldsymbol{\jmath}_{s}\right) \cdot \boldsymbol{\imath}_{0}\right), d_{y}=\operatorname{sign}\left(\left(\boldsymbol{\imath}_{s}+\delta \boldsymbol{\jmath}_{s}\right) \cdot \boldsymbol{\jmath}_{0}\right), T_{h}=T_{h}^{\min }$

with: $\delta=\operatorname{sign}\left(\mathbf{P P}_{\mathbf{s}} \cdot \jmath_{\mathbf{s}}\right)$, where $\mathbf{P} \mathbf{P}_{\mathbf{s}}$ is the vector from $\mathbf{P}$ to $\mathbf{P}_{\mathbf{s}}$.

Case 2: $r_{h, 2}<\rho_{k} \leq r_{h, 1}$ and $\gamma \cdot \boldsymbol{\imath}_{b}>0$. In this case the collision risk is more important and we set:

$$
\begin{aligned}
& d_{x}=\operatorname{sign}\left(\left(\boldsymbol{\iota}_{c}+\delta \boldsymbol{\jmath}_{c}\right) \cdot \boldsymbol{\imath}_{0}\right), d_{y}=\operatorname{sign}\left(\left(\boldsymbol{\iota}_{c}+\delta \boldsymbol{\jmath}_{c}\right) \cdot \boldsymbol{\jmath}_{0}\right) \\
& T_{h}=T_{h}^{\min }+\frac{T_{h}^{\max }-T_{h}^{\min }}{r_{h, 1}-r_{h, 2}}\left(r_{h, 1}-d_{k}\right)
\end{aligned}
$$

with: $\delta=\operatorname{sign}\left(\mathbf{P} \mathbf{P}_{\mathbf{s}} \cdot \jmath_{\mathbf{c}}\right)$, where $\mathbf{P} \mathbf{P}_{\mathbf{s}}$ is the vector from $\mathbf{P}$ to $\mathbf{P}_{\mathrm{s}}$.

Case 3: $\rho_{k} \leq r_{h, 2}$ and $\gamma \cdot \boldsymbol{\imath}_{b}>0$. In this case the collision risk is high and we set:

$d_{x}=\operatorname{sign}\left(\left(\boldsymbol{\imath}_{c}+\delta \boldsymbol{\jmath}_{c}\right) \cdot \boldsymbol{\imath}_{0}\right), d_{y}=\operatorname{sign}\left(\left(\boldsymbol{\imath}_{c}+\delta \boldsymbol{\jmath}_{c}\right) \cdot \boldsymbol{\jmath}_{0}\right), T_{h}=T_{h}^{\max }$

with: $\delta=\operatorname{sign}\left(\mathbf{P} \mathbf{P}_{\mathbf{s}} \cdot \jmath_{\mathbf{c}}\right)$, where $\mathbf{P} \mathbf{P}_{\mathbf{s}}$ is the vector from $\mathbf{P}$ to $\mathbf{P}_{\mathrm{s}}$. 


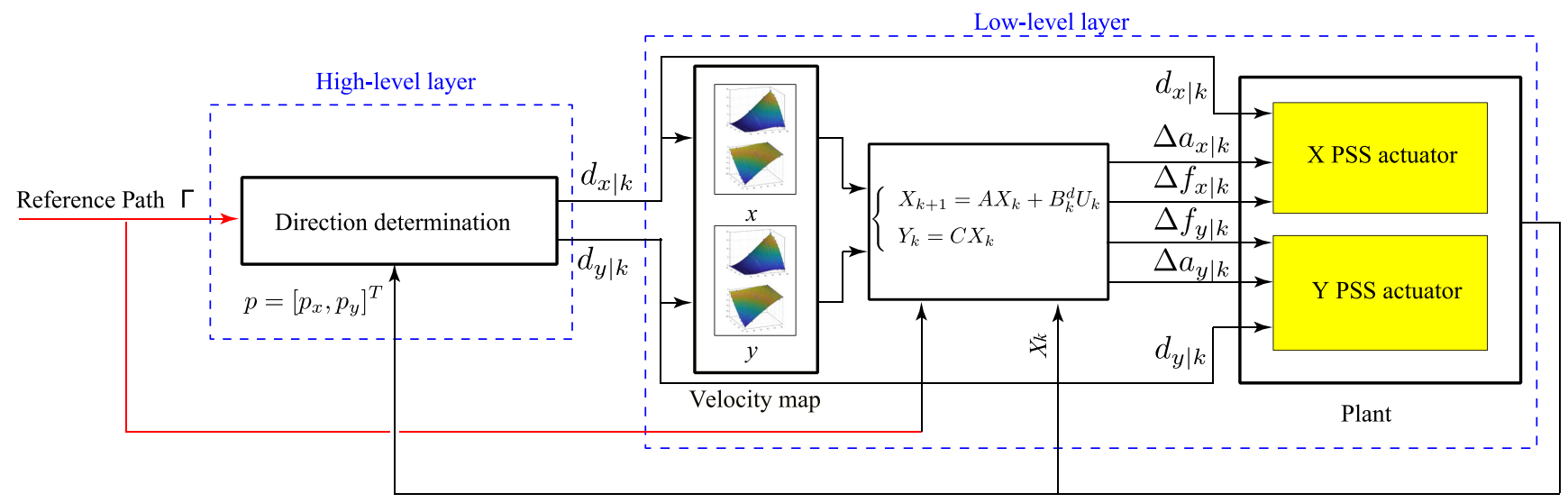

Fig. 4. Model predictive control (MPC)-based obstacle avoidance scheme. The high-level layer determines the direction. The low-level layer applies the control law.

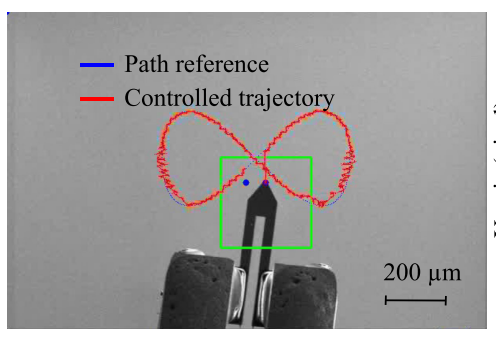

(a)

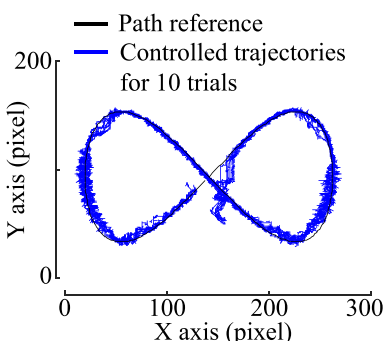

(b)

Fig. 5. Eight-shape reference MPC path following repeatability analysis with a specified velocity of $3 \mu \mathrm{m} / \mathrm{s}$ for 10 trials. (a) Path following result. (b) Repeatability results.

\section{EXPERIMENT}

Experiments focus on two aspects: the path following with a specified advancing velocity and the capability to avoid obstacles. The AFM probe position feedback is provided by Template Matching from SEM vision. qpOASES is used as the QP solver for MPC optimization.

The limit values for input and state constraints (see (13)-(14)) are defined as follows:

$$
\begin{aligned}
& U_{1, \text { min }}=U_{3, \text { min }}=-1 \mathrm{~V}, U_{1, \max }=U_{3, \max }=1 \mathrm{~V} \\
& U_{2, \min }=U_{4, \text { min }}=-5 \mathrm{~Hz}, U_{2, \max }=U_{4, \max }=5 \mathrm{~Hz} \\
& a_{x, \text { min }}=34 \mathrm{~V}, a_{y, \min }=30 \mathrm{~V}, a_{x, \max }=a_{y, \max }=80 \mathrm{~V} \\
& f_{x, \text { min }}=f_{y, \text { min }}=5 \mathrm{~Hz}, f_{x, \text { max }}=f_{y, \text { max }}=20 \mathrm{~Hz}
\end{aligned}
$$

The values of $T_{h}^{\min }, T_{h}^{\max }$ are defined as $2 s$ and $8 s$ respectively.

\section{A. Path Following by Model Predictive Control}

An eight-shape curve is defined as the reference path, which is characterized with varying curvatures and different motion directions in order to comprehensively and representatively test the tracking performance of the proposed algorithm. The aim of this experiment is to control the AFM probe to track the defined path with a specified advancing velocity.

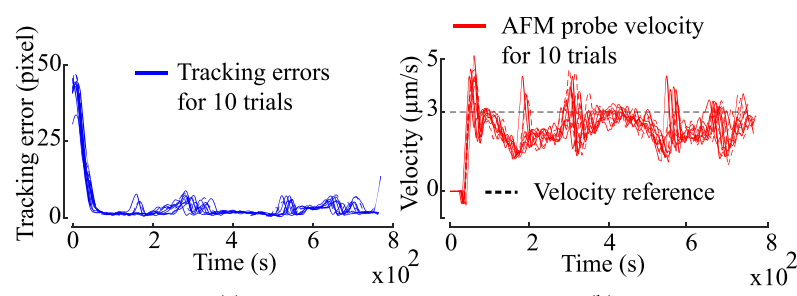

(a)

(b)

Fig. 6. Eight-shape reference MPC path following performance. (a) Tracking errors for 10 trials. (b) AFM probe velocity for 10 trials.

The tracking result is presented in Fig. 5(a). It can be found that the proposed algorithm is able to tune the AFM probe to track the defined reference path with various curvatures in various directions. The tracking repeatability is carried out by applying path following control on the AFM probe from the same initial location for 10 trials, as shown in Fig. 5(b). The AFM probe performs better when tracking the partial path with less curvature, and shortcuts more when it meets sharp turns. In addition, it vibrates more when moving on y axis direction compared with $\mathrm{x}$ axis direction. The vibrations can have several origins. The main probable raison is that at the corners of the path in Fig. 5, the velocity in y direction is close to 0 . The path distance where the velocity is close to 0 is longer in y axis compared to $\mathrm{x}$ axis. The lowest the velocity of a PIA actuator, the highest are the amplitude of the vibration. The latter is caused by the presliding dynamics of the slip motion [18]. One possible solution is to increase the frequency of the sawtooth signal to speed up the motion of the actuator (increasing velocity) and to minimize the duration where the velocity should be close to zero. However, this solution must be done considering the limitation of the SEM image acquisition rate for vision-based control.

Fig. 6 presents the position tracking error result (Fig. 6(a)) and the velocity tracking result (Fig. 6(b)), respectively. The position tracking error curve of Fig. 6(a) illustrates the convergences of the AFM probe to the eight-shape curve. There are fluctuations of the position tracking error curve because of the vibrations during the motion on y axis direction and sharp turns. On the other hand, the velocity control result of Fig. 6(b) shows that the actual AFM probe velocity fluctuates around the desired velocity, i.e. $3 \mu \mathrm{m} / \mathrm{s}$. It decreases at the sharp turns. 


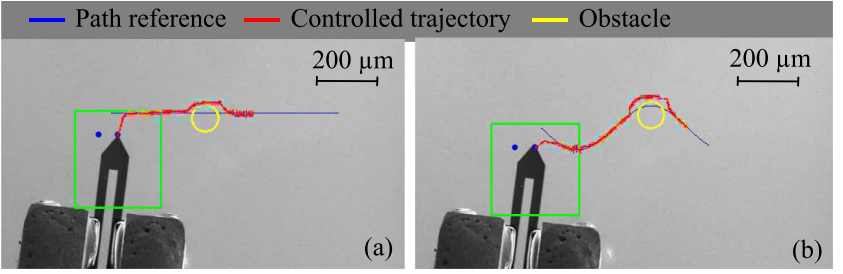

Fig. 7. Obstacle avoidance experimental results. The obstacle is shown in yellow. (a) Tracking results for a straight line shape reference path. (b) Tracking results for a sinusoidal shape reference path.
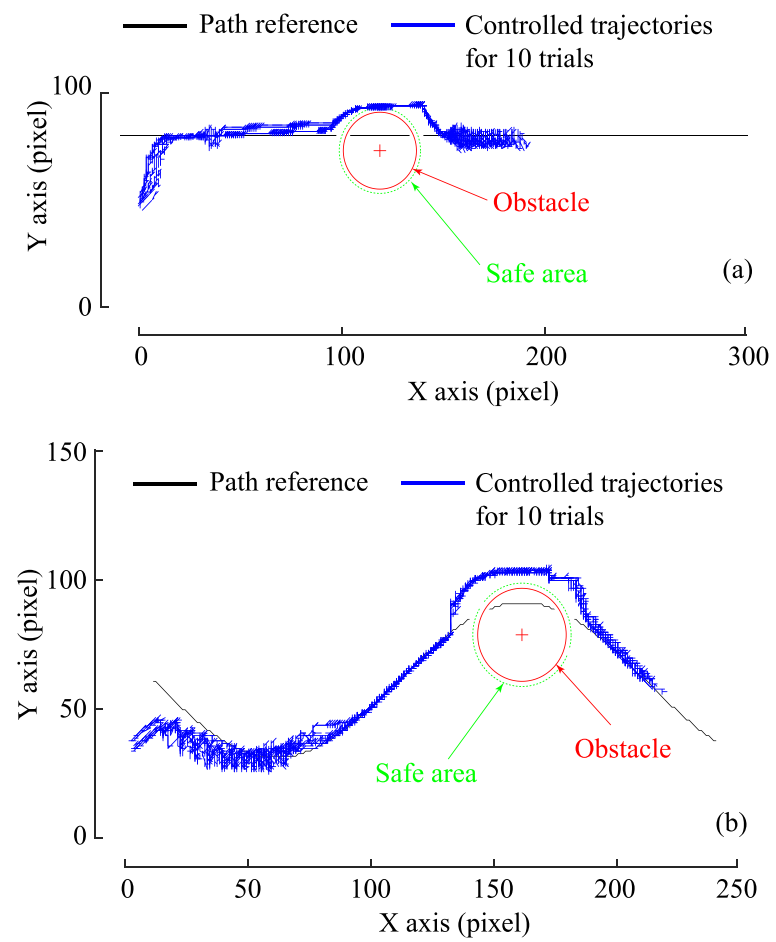

Fig. 8. Obstacle avoidance repeatability. (a) Controlled trajectories for 10 trials with a straight line reference path. (b) Controlled trajectories for 10 trials with a sinusoidal reference path.
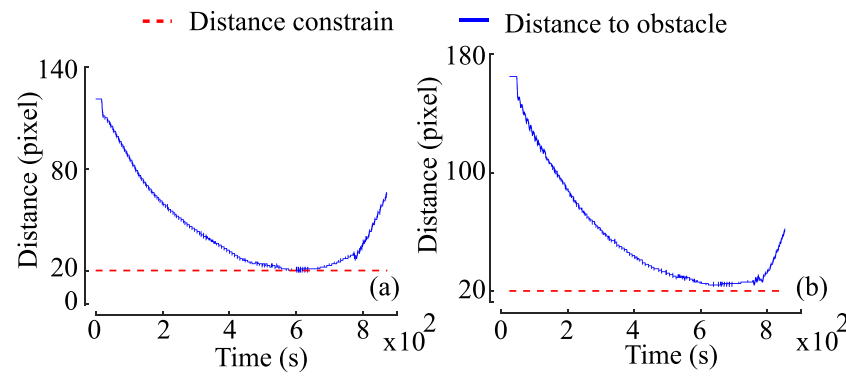

Fig. 9. Distance $\rho$ between the AFM probe tip and the obstacle during the obstacle avoidance. (a) Straight line reference path. (b) Sinusoidal reference path.
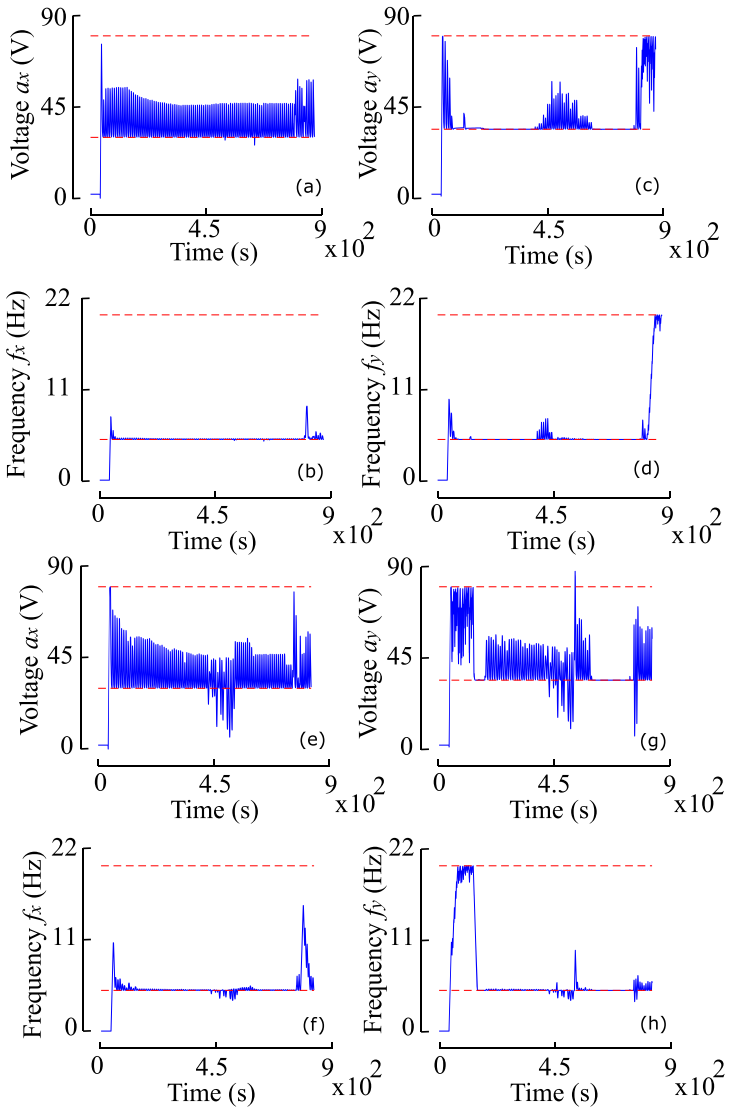

Fig. 10. PIA sawtooth input values. Subplots (a)-(d): obstacle avoidance with straight line reference path. Subplots (e)-(h): obstacle avoidance with sinusoidal reference path. Input constraints are defined by (15).

inside SEM. For objects whose dimensions is $100 \mu \mathrm{m}$ and more (for instance spherical objects with $100 \mu \mathrm{m}$ diameter), the path following with a mean error of $2.5 \mu \mathrm{m}$ is considered enough. In addition, it is possible to see in Fig. 6(a) that the maximum error in the worst case is lower than $10 \mu \mathrm{m}$. It is possible to reduce significantly this error by reducing the vibrations of PIA during the path following control. Indeed, due to the limited frequency rate of the SEM, we have limited the advancing velocity to $3 \mu \mathrm{m} / \mathrm{s}$. At this speed, the slip effect (let us recall that the PIA works with a stick and slip principle) generates vibrations. If the actuator is controlled at a higher speed, by increasing the frequency of the sawtooth voltage, the motion of the PIA will be smoother and therefore the mean error of the path following will be reduced. The reader can refer to our previous work [23] related to the dynamic control of PIAs.

\section{B. Obstacle Avoidance by Model Predictive Control}

A virtual obstacle is introduced along the reference path $\Gamma$. The obstacle is a circle of radius $r_{c}=18$ pixels, and we set $r_{s}=20$ pixels. The aim is to control the AFM probe tip to follow the curve $\Gamma$ and simultaneously avoid the virtual obstacle. The control procedure is executed under the previously defined input constraints. The tracking result is shown on Fig. 7. A straight line shape reference path and a sinusoidal shape reference path are used in Fig. 7(a) and Fig. 7(b) respectively. In both cases the AFM probe tip succeeds in converging to the path and bypassing the obstacle.
373 
To prove the repeatability, ten trials of obstacle avoidance controls are executed with both straight line and sinusoidal reference paths. Fig. 8 demonstrates that the obstacle avoidance behavior under the proposed algorithm is experimentally repeatable.

Fig. 9 shows the evolution of the distance $\rho$ from the AFM probe tip to the center of the obstacle. The state constraint $\rho \geq 20$ pixel is always satisfied.

Fig. 10 presents the PIA sawtooth input values, i.e., the amplitudes and the frequencies $a_{x}, f_{x}, a_{y}$ and $f_{y}$. The constraints for these variables are dfined by (15). The input constraints are globally well respected, with some deviations for the sinusoidal reference path.

The basic performances (step response and frequency characteristics of $\mathrm{X}$ and $\mathrm{Y}$ axis) are those of the low level control of the piezoelectric stick slip actuators (Fig. 4). In a previous work [23], [24], we have presented a detailed study about the basic performances of such actuators in open loop and closed loop. Considering the global MPC controller, the frequency of the sawtooth signal is limited to $20 \mathrm{~Hz}$. The velocity is $3 \mu \mathrm{m} / \mathrm{s}$. Therefore, for a reference position of $100 \mu \mathrm{m}$, the response time is $33 \mathrm{~s}$.

\section{CONCLUSION}

In this letter, a Model Predictive Control (MPC) has been formulated considering the specific working principle of piezoelectric inertia actuators (PIA) driven by a sawtooth type voltage. For that purpose, the idea has been to define a velocity map of such actuators which has been used as a reference model for MPC. A complete procedure for the synthesis of MPC considering such kind of actuators has been proposed. The controller has been applied for the specific case of path following and obstacle avoidance of an AFM probe held by PIAs and operating inside a SEM. The developed technique enriches the motion capability of nano-robotic systems operating inside SEM, accelerating the process towards a fully-automation for robotic manipulation purposes at small scales.

\section{REFERENCES}

[1] C. Shi et al., "Recent advances in nanorobotic manipulation inside scanning electron microscopes," Microsystems Nanoengineering, vol. 2, 2016, Art. no. 16024

[2] C. Ru et al., "Automated four-point probe measurement of nanowires inside a scanning electron microscope," IEEE Trans. Nanotechnol., vol. 10, no. 4, pp. 674-681, Jul. 2011

[3] J. Abrahamians, B. Sauvet, J. Polesel-Maris, R. Braive, and S. Régnier, "A nanorobotic system for in situ stiffness measurements on membranes," IEEE Trans. Robot., vol. 30, no. 1, pp. 119-124, Feb. 2014.

[4] S. Zimmermann, T. Tiemerding, O. C. Haenssler, and S. Fatikow, "Automated robotic manipulation of individual sub-micro particles using a dual probe setup inside the scanning electron microscope," in Proc. IEEE Int. Conf. Robot. Autom., 2015, pp. 950-955.

[5] M. Bartenwerfer et al., "Design of a micro-cartridge system for the robotic assembly of exchangeable afm-probe tips," in Proc. IEEE Int. Conf. Robot. Autom., 2013, pp. 1730-1735.
[6] J. Chen et al., "Automated sem-guided afm scan with dynamically varied scan speed," in Proc. Int. Conf. Manipulation, Autom. Robot. Small Scales, 2018.

[7] A. Bolopion, H. Xie, D. S. Haliyo, and S. Régnier, "Haptic teleoperation for 3-D microassembly of spherical objects," IEEE/ASME Trans. Mechatronics, vol. 17, no. 1, pp. 116-127, Feb. 2010.

[8] S. Fatikow, V. Eichhorn, T. Wich, T. Sievers, O. Hänßler, and K. N. Andersen, "Depth-detection methods for CNT manipulation and characterization in a scanning electron microscope," in Proc. Int. Conf. Mechatronics Autom., 2007.

[9] M. Ammi and A. Ferreira, "Robotic assisted micromanipulation system using virtual fixtures and metaphors," in Proc. IEEE Int. Conf. Robot. Autom., 2007, pp. 454-460.

[10] J.-O. Abrahamians, B. Sauvet, J. Polesel-Maris, R. Braive, and S. Régnier, "A nanorobotic system for in situ stiffness measurements on membranes," IEEE Trans. Robot., vol. 30, no. 1, pp. 119-124, Feb. 2014

[11] V. Eichhorn et al., "Nanolab: A nanorobotic system for automated pickand-place handling and characterization of cnts," in Proc. IEEE Int. Conf. Robot. Autom., 2009, pp. 1826-1831.

[12] X. Ye, Y. Zhang, C. Ru, J. Luo, S. Xie, and Y. Sun, "Automated pickplace of silicon nanowires," IEEE Trans. Autom. Sci. Eng., vol. 10, no. 3 , pp. 554-561, Jul. 2013.

[13] Q. Shi et al., "A vision-based automated manipulation system for the pick-up of carbon nanotubes," IEEE/ASME Trans. Mechatronics, vol. 22, no. 2, pp. 845-854, Apr. 2017

[14] Y. Guo et al., "Automated pick-up of carbon nanotubes inside a scanning electron microscope," in Proc. IEEE/RSJ Int. Conf. Intell. Robots Syst., 2016, pp. 5318-5323.

[15] C. Zhou et al., "A closed-loop controlled nanomanipulation system for probing nanostructures inside scanning electron microscopes," IEEE/ASME Trans. Mechatronics, vol. 21, no. 3, pp. 1233-1241, Jun. 2016.

[16] B. Mokaberi, J. Yun, M. Wang, and A. A. Requicha, "Automated nanomanipulation with atomic force microscopes," in Proc. IEEE Int. Conf. Robot. Autom., 2007, pp. 1406-1412.

[17] U. Mick, V. Eichhorn, T. Wortmann, C. Diederichs, and S. Fatikow, "Combined nanorobotic afm/sem system as novel toolbox for automated hybrid analysis and manipulation of nanoscale objects," in Proc. IEEE Int. Conf. Robot. Autom., 2010, pp. 4088-4093.

[18] M. Boudaoud, T. Lu, S. Liang, R. Oubellil, and S. Régnier, "A voltage/frequency modeling for a multi-dofs serial nanorobotic system based on piezoelectric inertial actuators," IEEE/ASME Trans. Mechatronics, vol. 23 , no. 6 , pp. $2814-2824$, Dec. 2018.

[19] T. Lu, M. Boudaoud, D. Hériban, and S. Régnier, "Nonlinear modeling for a class of nano-robotic systems using piezoelectric stick-slip actuators," in Proc. IEEE/RSJ Int. Conf. Intell. Robots Syst., 2015, pp. 6020-6025.

[20] W. Rong, S. Liang, L. Wang, S. Zhang, and W. Zhang, "Model and control of a compact long-travel accurate-manipulation platform," IEEE/ASME Trans. Mechatronics, vol. 22, no. 1, pp. 402-411, Feb. 2017.

[21] S. Liang, M. Boudaoud, B. Cagneau, and S. Régnier, "Velocity characterization and control strategies for nano-robotic systems based on piezoelectric stick-slip actuators," in Proc. IEEE Int. Conf. Robot. Autom., 2017, pp. 6606-6611.

[22] B. Gutjahr, L. Gröll, and M. Werling, "Lateral vehicle trajectory optimization using constrained linear time-varying mpc," IEEE Trans. Intell. Transp. Syst., vol. 18, no. 6, pp. 1586-1595, Jun. 2017.

[23] R. Oubellil, A. Voda, M. Boudaoud, and S. Régnier, "Mixed stepping/scanning mode control of stick-slip sem-integrated nano-robotic systems," Sensors Actuators A: Physical, vol. 285, pp. 258-268, 2019.

[24] R. Oubellil, A. Voda, M. Boudaoud, and S. Régnier, "A 2-DOF h control strategy for a 3 axes robotic system operating at the nanometer scale," in Proc. Int. Conf. Syst. Theory, Control Comput., 2016. 